\title{
Addressing Systematic Uncertainties in Black Hole Mass Measurements
}

\author{
Kelly D. Denney ${ }^{* a}{ }^{a}$ R. J. Assef, ${ }^{b}$ M. C. Bentz,${ }^{c}$ M. Dietrich, ${ }^{d}$ K. Horne, ${ }^{e}$ \\ C. S. Kochanek, ${ }^{d}$ S. Mathur, ${ }^{d}$ B. M. Peterson, ${ }^{d}$ R. W. Pogge, ${ }^{d}$ and M. Vestergaard ${ }^{a}$ \\ ${ }^{a}$ DARK Fellow, Dark Cosmology Centre \\ Niels Bohr Institute, University of Copenhagen, Copenhagen, Denmark \\ ${ }^{b}$ NASA Postdoctoral Program Fellow, Jet Propulsion Laboratory \\ California Institute of Technology, Pasadena, CA, USA \\ ${ }^{c}$ Georgia State University, Atlanta, GA, USA \\ ${ }^{d}$ University of St. Andrews, St. Andrews, Scotland, UK \\ ${ }^{e}$ The Ohio State University, Columbus, OH, USA \\ E-mail: kelly@dark-cosmology.dk
}

\begin{abstract}
We present selected efforts underway to mitigate systematic uncertainties in supermassive black hole (BH) mass estimates. First, we highlight a NLS1-sensitive systematic in determining Balmer-based single-epoch BH masses, where line width measurements may be biased due to contamination from narrow emission-line components. Next, we present recent, detailed reverberation mapping results by showcasing a velocity-delay map of the NLS1 galaxy NGC 4051. This visualization of the transfer function describing the emission line response of the broad line region (BLR) gas to variations in the continuum emission shows the kinematics and geometry of the BLR in velocity and time-domain space. These maps will help to constrain the geometric scale factor, $\langle f\rangle$, that is currently the largest source of systematic uncertainty in direct, reverberationbased BH mass measurements. Finally, we show that single-epoch C IV-based BH mass estimates are consistent with their Balmer-based counterparts when efforts are made to use only high $S / N$ data and a homogeneous prescription for line width and luminosity measurements and to correct an observed color dependence in the C IV-to-Balmer mass ratio residuals.
\end{abstract}

Narrow-Line Seyfert 1 Galaxies and their place in the Universe - NLS1,

April 04-06, 2011

Milan Italy

${ }^{*}$ Speaker. 


\section{Introduction}

The well-accepted paradigm that all massive galaxies house supermassive black holes (BHs) at their centers and that accretion onto such BHs is synonymous with AGN emission has landed the study of AGNs and supermassive black holes at the forefront of astrophysical research. Not only do these BHs exist in the centers of galaxies, but evidence suggests they co-evolve with their hosts and influence their environment on larger scales than the direct reach of their gravitational potential (e.g., Ferrarese \& Merritt, 2000; Gebhardt et al., 2000; Marconi \& Hunt, 2003; Häring \& Rix, 2004; Hopkins \& Elvis, 2010; Debuhr, Quataert, \& Ma, 2011, though see also Peng 2007; Jahnke \& Maccio, 2010). Studying galaxy evolution across cosmic time and the connection to the $\mathrm{BHs}$ that lie at their centers necessarily involves a census of the demographics of these black holes. Thus, a fundamental property of the BHs to investigate is their mass.

Black hole masses can be measured directly using (1) the dynamical motions of gas and stars in the central regions of nearby quiescent galaxies where the sphere of influence of the BH is spatially resolved (see Gültekin et al., 2009, and references therein), (2) $\mathrm{H}_{2} 0$ megamasers in Type 2, or narrow-line, AGNs (e.g., Moran et al., 1995; Kuo et al., 2011), and (3) reverberation mapping in Type 1 AGNs (Blandford \& McKee, 1982; Peterson 1993, see also Section 3). All of these methods are currently only useable for relatively nearby sources due either to technological constraints (e.g., angular resolution) or observational feasibility (e.g., temporal resolution of RM campaigns). This makes direct measurement of black holes outside the local universe an unrealistic goal, at present.

Fortunately, local direct mass measurements have led to the discovery of empirical scaling relationships between the observed properties of BHs, AGNs, and their host galaxies. Of particular importance here is the $R_{\mathrm{BLR}}-L$ relationship for Type 1 AGNs (e.g., Kaspi et al., 2000; Bentz et al., 2009a), which shows a tight correlation between the monochromatic continuum luminosity of an AGN and the characteristic radius of emission from its broad line region (BLR), which is typically calibrated with $\mathrm{H} \beta$ (see work by $\mathrm{M}$. Bentz, these proceedings). The origin of this relation is linked to the photoionization physics responsible for the BLR gas emission, and the most current empirically-calibrated slope of Bentz et al. is consistent with the slope naively predicted under the assumption of a self-similar model for all AGNs. This scaling relationship allows for the calculation of single-epoch virial $\mathrm{BH}$ masses: $M_{\mathrm{BH}}=f R_{\mathrm{BLR}} V^{2} / G$, where $R_{\mathrm{BLR}}$ is determined by using the luminosity measured from the continuum region of the single-epoch spectrum as a proxy for the BLR radius with the $R_{\mathrm{BLR}}-L$ relationship, $V$ is estimated from a measure of the Dopplerbroadened broad emission-line width, $f$ is a scale factor of order unity and that depends on the BLR geometry and kinematics (discussed in more detail in Section 3), and $G$ is the gravitational constant. By calculating BH masses indirectly in this way, BHs can be studied in AGNs at all redshifts and for large spectroscopic samples of objects, such as those coming out of the Sloan Digital Sky Survey (SDSS).

\section{Systematic Uncertainties in Single-Epoch Masses}

While single-epoch mass measurements are a simple and effective method for studying black hole masses and, thus, the connection between BHs and galaxy evolution at all redshifts, there are many systematic uncertainties to be aware of when applying this method to any number of spectra. 
We have investigated several systematic uncertainties that affect single-epoch $\mathrm{BH}$ mass measurements (see Denney et al., 2009a). These include contributions from AGN variability, host galaxy contamination to the continuum luminosity, narrow-line contamination to the line width measurements, data quality (i.e., $S / N$ ), and blending of spectral features. Each of these systematics that affects the line width measurement is investigated with respect to two common ways of characterizing the line width: the FWHM and the line dispersion (i.e., second moment of the line profile), $\sigma_{\text {line }}$. Understanding and mitigating systematic uncertainties in these seemingly simple mass calculations is important because interpreting results of BH mass measurements of large samples of objects in the presence of systematics could lead to, e.g., misunderstandings of the physical properties of these objects and their role in cosmic evolution (see, e.g., Rafiee \& Hall, 2011). While all of the sources of uncertainty we discuss in Denney et al. (2009) should be considered in the calculation of single-epoch BH masses, we highlight here (1) the significance of narrow line contamination in estimates of BH masses of NLS1s and (2) the importance of obtaining high $S / N$ data for these types of studies in all AGNs.

\subsection{NLS1 Sensitive Systematics}

Single-epoch BH mass determinations of NLS1s are particularly sensitive to contamination from line emission originating in the narrow-line region (NLR). This emission comes from low density gas on kpc scales in the AGN, and is therefore considered a constant flux component (i.e., it doesn't reverberate) whose velocity field is not directly under the influence of the gravity of the BH. In this case, this blended emission should be subtracted from the broad-line profiles before the line width is measured. Failure to subtract narrow-line emission from the line profile leads to a systematic underestimate of the line width. As the line width enters the BH mass equation to the second power, this can lead to a significant underestimate of the BH mass. By comparing the mean of the distribution of virial products ${ }^{1}$ in each top panel of Figure 1 to the respective bottom panel, we show that this can affect the mean virial product by as much as an order of magnitude when the FWHM is used to characterize the line width (right panels) and the narrow-line component is prominent in the line profile (such as for NGC 5548). This systematic underestimate of the masses is much smaller when characterizing the width using the line dispersion (left panels). Nonetheless, making accurate mass measurements requires that this narrow-line contamination be removed. Unfortunately, the exact contribution of narrow-line flux varies from object to object, as does the magnitude of the systematic effect due to the large variety of broad-line profile shapes. This makes it impossible to determine a global estimate of the impact of this systematic on a sample of objects, making it necessary to correct individual objects before the line widths are measured.

The reason the effects of this particular systematic are important to recognize in connection with NLS1s is that it is difficult to take into account and subtract the narrow-line component from NLS1 emission-line profiles. In BLS1s, the narrow-line component is often clearly distinct from the broad-line component, making subtraction relatively simple. However, as illustrated by the $\mathrm{H} \beta$ profile of four reverberation mapped NLS1s in Figure 2, the superposed narrow-line components are clearly not an obvious and prominent feature as is seen in some AGNs. In some ways, this

\footnotetext{
${ }^{1}$ The virial product, i.e., $M_{\mathrm{BH}} / f$ is used here because all measurements are from a single object, and thus a single black hole mass. This quanitity differs with line width characterization because the absolute AGN mass scale will have a different calibrating factor of $f$ for FWHM vs. the line dispersion.
} 

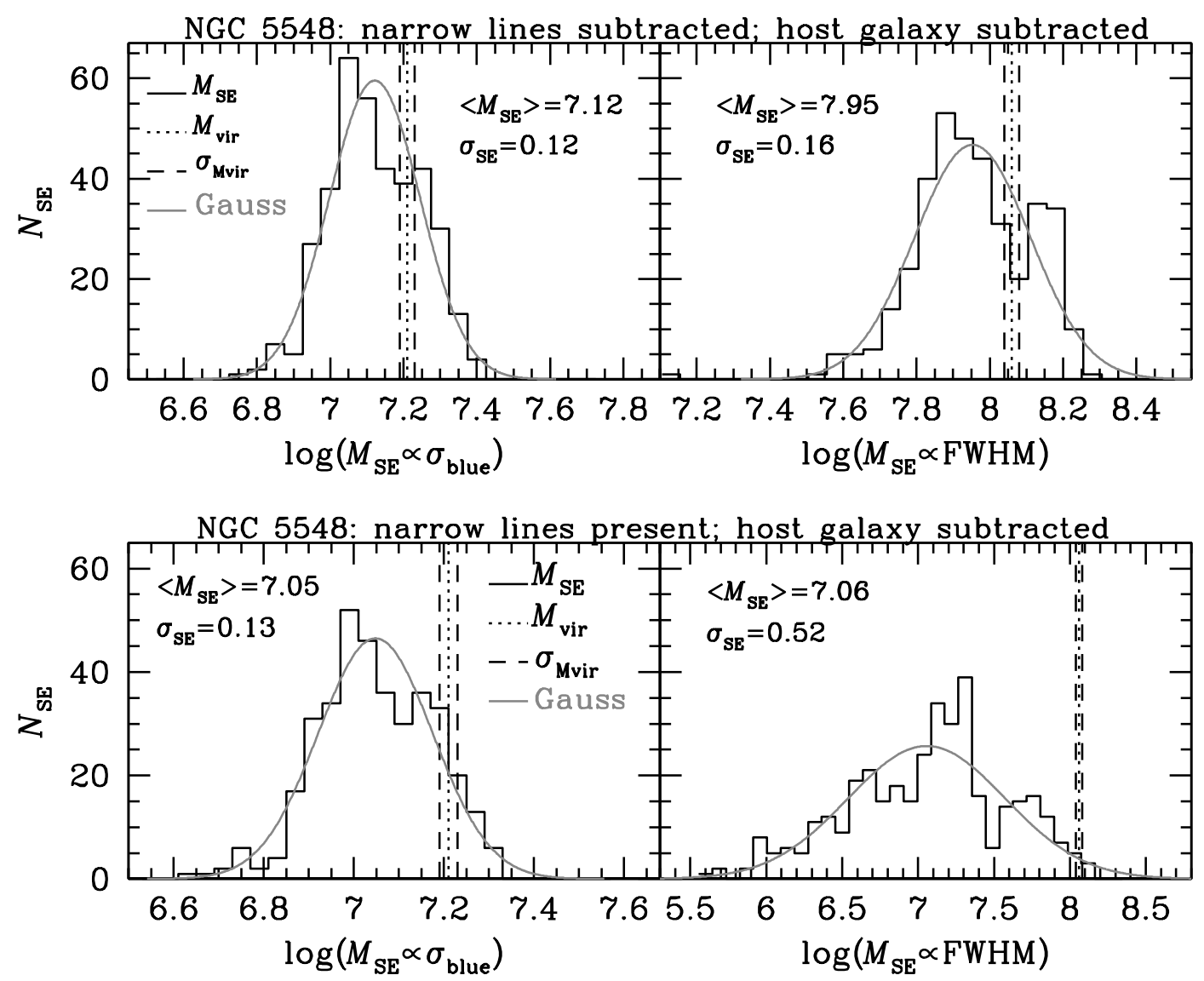

Figure 1: Virial product (VP) distributions (i.e., $V P=M_{\mathrm{BH}} / f$ ) for NGC 5548; reproduced from Denney et al. (2009a). Solid lines show the distributions of VPs calculated using both the line dispersion (left) and the FWHM (right) to measure $\mathrm{H} \beta$ widths. A Gaussian function with the same mean, dispersion, and area as the data is over-plotted in gray. The vertical lines represent the reverberation VP (dotted) and measurement uncertainties (dashed). Top: Narrow lines and host galaxy starlight have been subtracted from all spectra before calculating VPs. Bottom: galaxy starlight has been subtracted, but the narrow lines were left in the spectra before measuring the line widths. The distribution mean and dispersion are shown in each panel. The systematic shift in the mass distributions between the top and bottom panels demonstrates how masses are systematically underestimated when the narrow-line component is not subtracted.

reduces the biases created by the narrow lines, compared to the example in Figure 1. However, the smooth transitions from the broad components to any narrow components make it difficult, if not impossible, to reliably separate the contribution from each emission-line component. Since AGN broad emission lines do not have any expected shape, it is difficult to justify a priori what the broadline profile should be. Based on near-IR observations of Paschen series emission lines, Landt et al. (these proceedings) have suggested that the broad-lines are intrinsically flat-topped, indicative of emission arising from a disk with a finite outer radius. The full line profile then has a Gaussianlike shape only after the narrow-line is superposed on top of the flat-topped broad-line profile. If true, this could provide a constraint on the amount of narrow-emission to subtract. However, many BLS1 profiles from which the narrow-line components can be reliably subtracted do not have such 


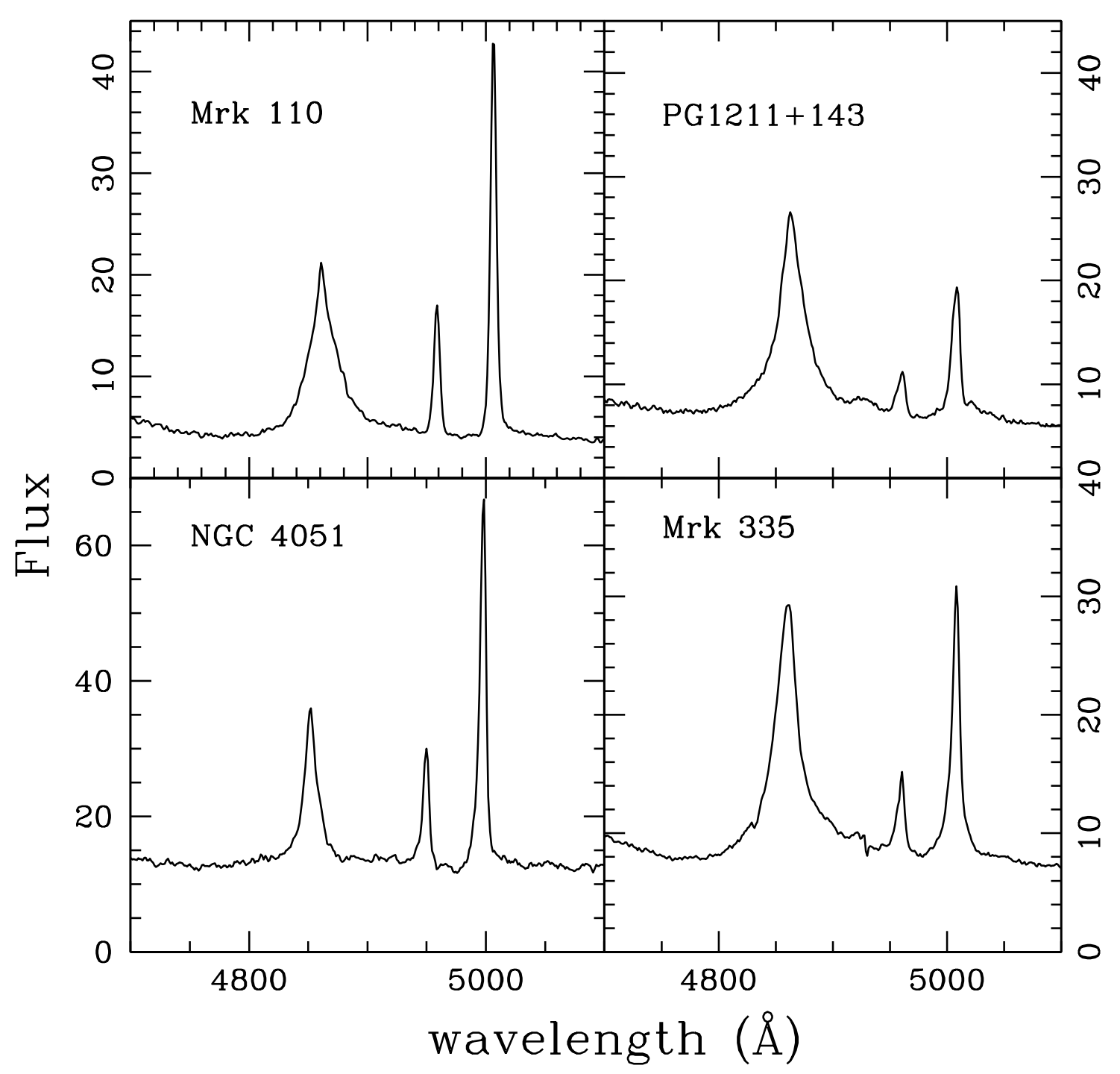

Figure 2: Example $\mathrm{H} \beta$ spectra of four NLS1 galaxies.

flat tops, so clearly more work in needed to try to better understand these blended line components.

Since failure to account for the narrow-line contribution will lead to an underestimate of the line width and resulting single-epoch mass estimate ${ }^{2}$, awareness of this trend is particularly relevant for NLS1 mass estimates. NLS1s have been interpreted to be objects with high accretion rates and small black hole masses for their luminosities (e.g., Mathur, 2000). Therefore is it important to try to keep this systematic effect, which would result in artificially low mass measurements in NLS1s, from biasing the evidence suggesting that objects in this class truly have small black hole masses.

\footnotetext{
${ }^{2}$ Reverberation-based masses are not susceptible to narrow-line contamination because they are based on line widths measured from the rms, or variable emission, spectrum, to which narrow-lines do not contribute.
} 


\subsection{Data Quality Considerations}

The accuracy and reliability of single-epoch black hole mass estimates are significantly affected by the quality of the data used for making the line width measurements, and simply "beating down" the uncertainties with large number statistics does not always work in these instances. Denney et al. (2009a) discuss severals ways that systematic uncertainties can be introduced in BH mass measurements made using low $S / N$ data. Here we focus on the introduction of uncertainties in the masses due to the possible presence of undetected absorption in the line profile. Absorption comes in many shapes, sizes, and depths, and can easily be hidden in a low $S / N$ spectrum. In some cases the majority of the flux on the blue side of a line profile, particularly C IV $\lambda 1549$ can be absorbed (see additional discussion on C IV in Section 4), but the presence of this absorption can be masked by poor data quality. If the absorption is not recognized, line widths and thus $\mathrm{BH}$ masses can be systematically and significantly underestimated. Figure 3 shows an example of this scenario. The top panel shows the original spectrum of a lensed quasar, SDSS1151+0340, observed as part of the SDSS. There does not seem to be any obvious indication of absorption in the blue side of the C IV profile; instead, the spectrum blueward of C IV simply appears to be a noisy continuum, as expected with the $S / N$ measured in the continuum near $1450 \AA$ to be $2.0 \mathrm{pixel}^{-1}$. However, Netzer et al. (2007) found that the C IV BH mass of this object is underestimated compared to that derived from $\mathrm{H} \beta$ by a factor of almost 6 , making it one of the largest outliers in their Figure 4 . We re-observed this object at both MDM and Palomar Observatories (Figure 3, middle and bottom, respectively; see Assef et al., 2011, for details). Unfortunately, poor observing conditions prevented a significant increase in the $S / N$ of our new spectra. Nonetheless, certainly one and possibly two absorption troughs are present in the blue side of the C IV line, explaining the large underestimate of the $\mathrm{C}$ IV versus $\mathrm{H} \beta \mathrm{BH}$ mass estimate for this object reported by Netzer et al.

There is no way to correct for underestimates in line width measurements due to absorption, when the absorption is hidden in the noise due to the poor quality and low $S / N$ of a spectrum. The only way to mitigate this type of systematic is to obtain higher $S / N$ spectra. We stronly recommend making SE BH measurements only with spectra with $S / N \gtrsim 20$ pixel $^{-1}$ in the continuum (see Denney, 2009a). With high quality spectra, one can recognize the presence of absorption and either mark the masses derived from such spectra as lower limits, or completely discard them from the sample. The SE method for estimating BH masses has become broadly applicable due to the use of automated pipelines that can apply this method to large samples of survey spectra. However, spectra in many such samples, particularly for faint sources outside the local universe, are often of lower quality due to the need to observe a vast number of objects in a limited amount of time. Therefore, it is imperative that the community be aware of and correct for such 'hidden' systematics that can skew the conclusions of such studies.

\section{Reverberation Mapping of NGC 4051}

Reverberation mapping takes advantage of a measurable time delay between variations in the continuum luminosity and those observed in the reprocessed emission from photoionized gas in the broad-line region (BLR). Light travel time arguments indicate that this delay, or lag, corresponds to the radius of the gas from the $\mathrm{BH}$. Combining this radius with BLR gas kinematics inferred from 


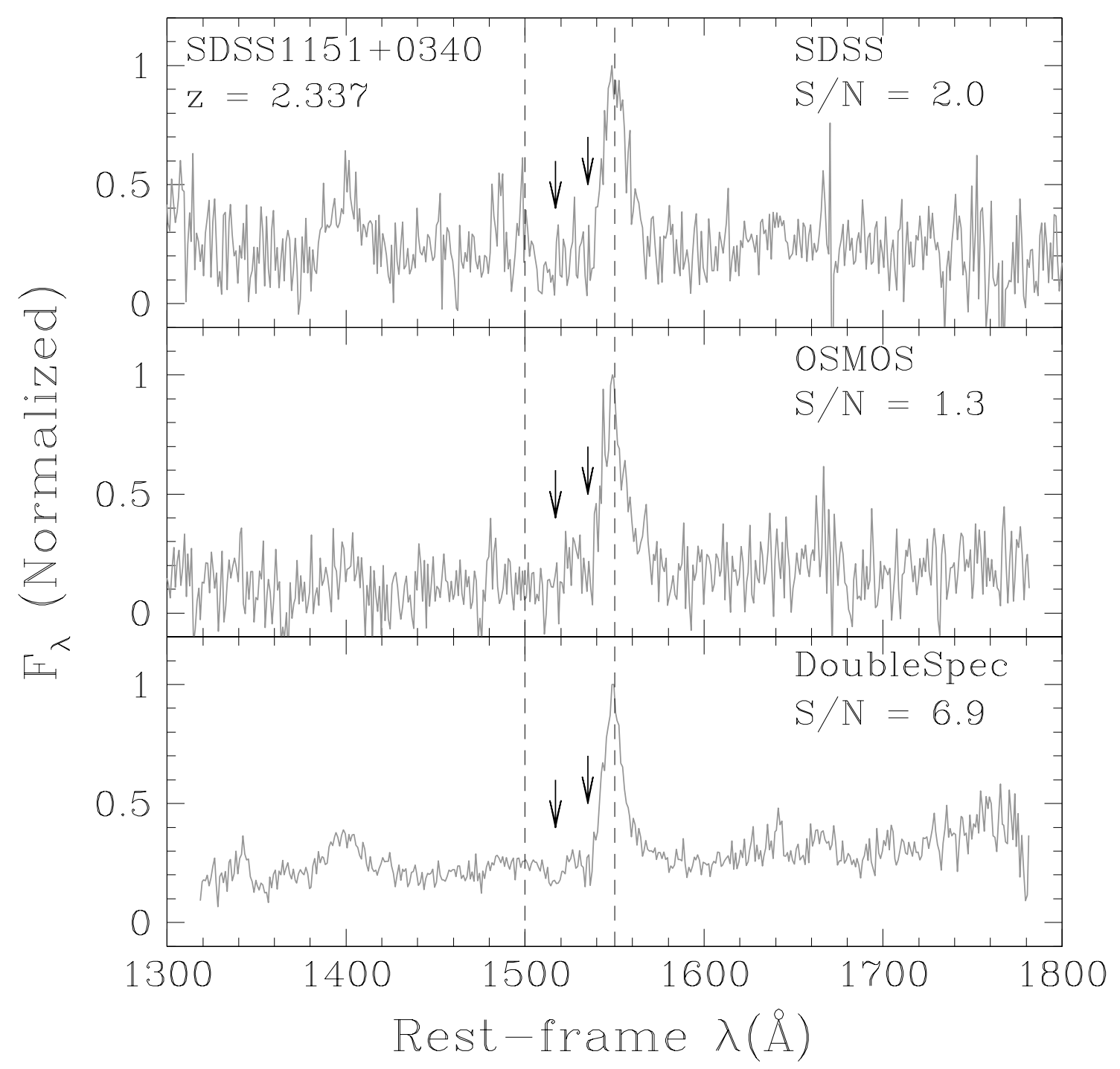

Figure 3: Three spectra of the lensed quasar SDSS1151 from SDSS (top), MDM Observatory (middle), and Palomar Observatory (bottom). The Palomar and MDM spectra show that absorption exists in on the blue side of the C IV line profile that was masked in the original SDSS spectrum due to the low $S / N$ of that spectrum.

the Doppler-broadened emission lines provides a means for measuring the $\mathrm{BH}$ mass by exploiting evidence for the virialization and gravitational binding of the BLR gas to the $\mathrm{BH}$, through the equation given above. Unlike SE masses, however, which use the luminosity as a proxy for the BLR radius, reverberation mapping measures this radius directly (and is thus the basis for calibrating the $R_{\mathrm{BLR}}-L$ relation). Using this prescription, black hole masses have been measured directly in more than four dozen AGNs (see Peterson et al., 2004; Bentz et al., 2009b; Denney et al., 2010). Despite the fact that these are direct measurements based on the line-of-sight (LOS) dynamics of the BLR gas, these measurements are still systematically uncertain due to the unknown geometry and 
kinematics of this gas. This uncertainty is accounted for in the mass determination with the virial scale factor, $f$. Currently, this factor is estimated statistically for the ensemble of RM mass measurements by assuming that AGNs follow the same $M_{\mathrm{BH}}-\sigma_{\star}$ relationship as quiescent galaxies, and the only difference is a zero-point offset in the AGN masses due to this geometric factor (see Onken et al., 2004; Woo et al., 2010). Woo et al. (2010) find that the largest source of systematic uncertainty in an individual RM measurement is due to object-to-object differences in the value of $f$. Therefore, the only way to significantly reduce the systematic uncertainties in RM masses is by better understanding the BLR. Luckily, RM methods provide a means for doing this, too. To date, RM has primarily been used to only first order, which results in a mean time delay, and thus radius, for the BLR. However, since the BLR is actually an extended region, there is not simply a single delay and velocity that describe the location and kinematics of the emitting gas. Instead, the response of the distribution of BLR gas to a continuum outburst is mathematically contained in the transfer function, which is a function of both LOS velocity and position, i.e., time-delay. We graphically reproduce this transfer function as a 2-dimensional "velocity-delay map" (VDM), and by comparing models and simulations of the BLR to observed VDMs, we can reconstruct the geometry and kinematics of the BLR (see Horne, 1994). This will allow for a direct measurement of $f$ for individual objects, and given enough individual measurements, constrain its ensemble average that is applied to all objects. Unfortunately, this higher-order RM analysis has stringent data requirements (Horne et al., 2004), and most attempts at this analysis have produced ambiguous results, at best. The most detailed VDM so far is that describing the BLR of Arp 151 from Balmer and He II $\lambda 4686$ emission (Bentz et al., 2010, see also Peterson et al., these proceedings).

We are working to produce VDMs for $\mathrm{H} \beta$ and He II reverberation observed during our 2007 RM campaign at MDM observatory (Denney et al., 2009b; Denney et al., 2009c; Denney et al., 2010) using the maximum entropy methods of Horne (1994). Figure 4 shows a preliminary VDM for the NLS1 NGC 4051. Unfortunately, even with the $\lesssim 1$ day temporal sampling intervals that we obtained during this campaign, the VDM still appears poorly resolved as a function of time delay. In particular, the mean delay of the He II $\lambda 4686$ emission appears to be on shorter time scales than our median sampling rates, indicating that higher sampling rates are needed in order to be able to measure the characteristic radius of the He II emitting region. Furthermore, although the mean response of the $\mathrm{H} \beta$ emission appears to be $\sim 2-3$ days, further resolution around this mean delay is also not possible with the current temporal resolution beyond an indication of both shorter and longer lags for the slowest moving gas whose emission appears toward the center of the line profile.

We have not yet fit BLR geometric and/or kinematic models to these VDM results, as our fits are not yet finalized. However, a simple visual inspection of this VDM can still reveal some probable characteristics of the geometry and kinematics of the BLR in NGC 4051. As mentioned above, the narrow line profiles observed in NLS1 optical spectra could be evidence for a truly small black hole mass; however, another explanation is that the broad-line profiles could be narrow simply because of inclination effects in a unified model of AGNs (cf., Antonucci, 1993, and Boroson et al., these proceedings). There is ample evidence in support of both explanations, and there are probably objects in this class due to both effects (afterall, NLS1s are strictly defined based only on their $\mathrm{H} \beta$ line width). NGC 4051, in particular, does not exhibit some of the typical characteristics of objects in the NLS1 class. For instance, it is not accreting at a high Eddington fraction (i.e., $L / L_{E d d}=0.030$ ) unlike many NLS1s. Furthermore, Fischer et al. (these proceedings; see 


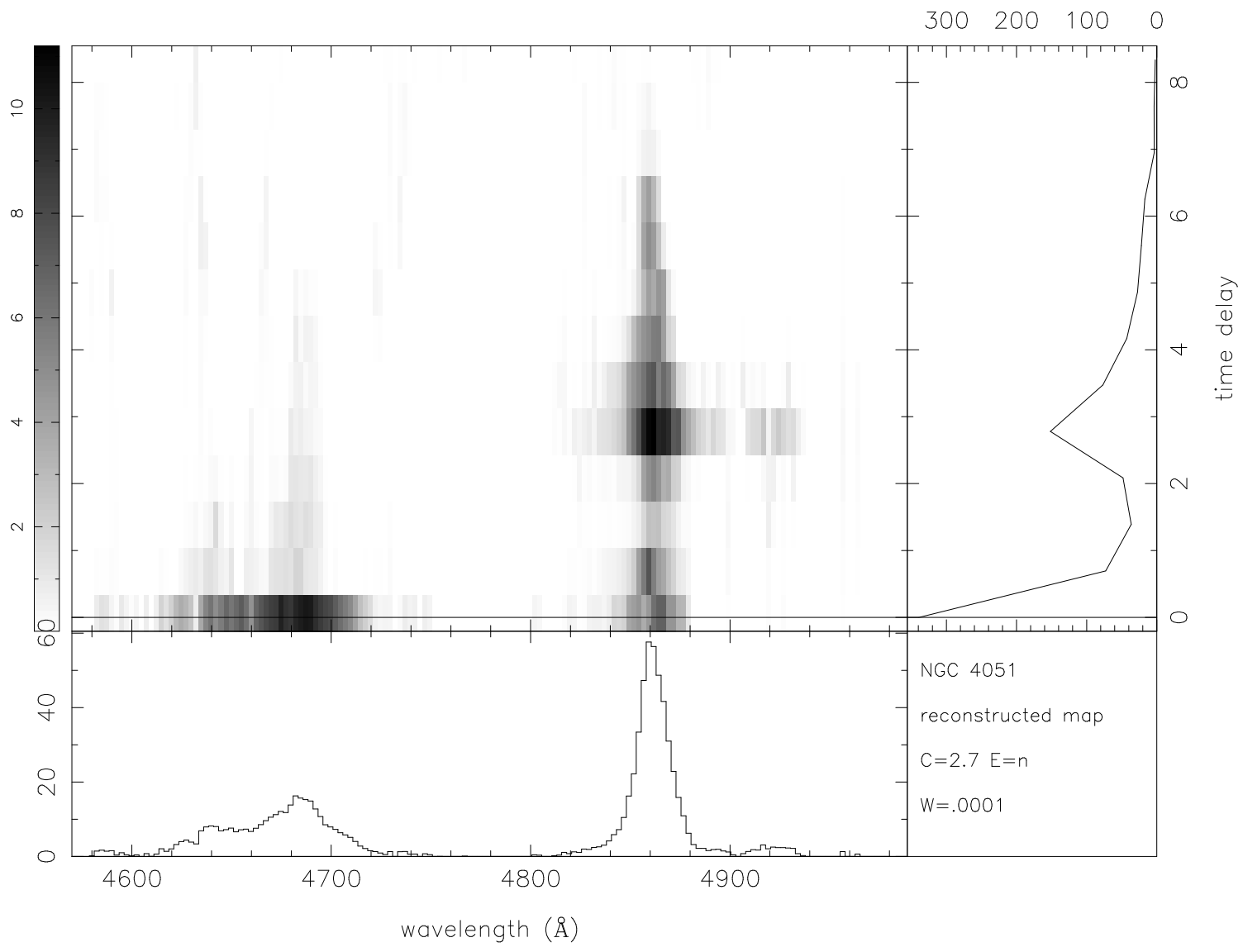

Figure 4: Preliminary velocity delay map of NGC 4051. The bottom left panel shows the variable flux as a function of wavelength, i.e., the time-delay-integrated response as a function of wavelength. The upper right panel shows the 1D transfer function, i.e., effectively the LOS velocity-integrated response of the emission line as a function of time delay. The central panel shows in gray scale the $2 \mathrm{D}$ velocity delay map: the observed emission-line response as a function of LOS velocity and time delay.

also Crenshaw et al., these proceedings) find evidence from NLR kinematics for NGC 4051 being oriented nearly pole-on. Does this picture fit with the information we can glean from the VDM in Figure 4? Aspects of the H $\beta$ RM signal in our VDM could certainly support the pole-on geometry. First, we see that most of the reverberation signal is coming from a very small range in lags (i.e., $\Delta \tau \sim 1$ day) across the entire line profile, consistent with looking at a nearly face-on distribution of gas. The $\mathrm{H} \beta$ VDM shows a weaker signal at longer lags for the lowest velocity gas, where this change in delay is relatively symmetric about the gas velocity distribution. This could be an indication of roughly Keplarian orbits in a disk-like geometry as well. However, there also appears to be low-velocity gas with zero lag, indicating that this gas is along the line of sight, but with no obvious net inflow or outflow velocity. It is not clear from simple visual inspection how this gas fits into the picture, but more detailed BLR models should lend to a unified picture based on these results.

Because the He II is still largely unresolved, it is difficult to say much about this line, except for two points. (1) Even unresolved, it is clear that the He II time delay is shorter than that of $\mathrm{H} \beta$, and the He II line width is also broader than $\mathrm{H} \beta$, signifying higher velocities in the He II emitting 
region. This further supports the picture of an ionization-stratified BLR. (2) The He II profile has a significantly blueshifted emission component, which has been previously noted by Peterson et al. (2000). Our VDM shows evidence that the time-delay distribution from this blueshifted component does not extend to lags as large as those seen in the core of the He II line. Since this blueshifted gas has, on average, shorter lags, this may indicate that some kinematic component of the He IIemitting region of the BLR is outflowing. As our analysis of these observations continues, we hope a comparison of these results to BLR models will be able to create a coherent picture of the geometry and kinematics of the BLR in NGC 4051. However, even models cannot constrain unresolved signals, indicating that more observations with even higher time sampling are needed to fully resolve and describe the BLR geometry and kinematics in this low-luminosity NLS1.

\section{C IV-based Masses}

Locally, where single-epoch mass scales can be calibrated against direct $\mathrm{BH}$ mass measurements as described above, these SE masses are typically estimated from the width of $\mathrm{H} \beta$. For AGNs at cosmological distances, however, this line is shifted out of the optical spectrum, and mass estimates are typically only obtained from the Mg II $\lambda 2798$ and C IV $\lambda 1549$ broad emission lines at intermediate and high redshift due to the difficulty of ground-based IR observations. There still exists considerable debate as to the reliability of using C IV to measure black hole masses. As C IV is a resonance line, it is highly susceptible to absorption. Furthermore, difficulties such as large observed blueshifts and profile asymmetries, possibly associated with outflows or winds (see, e.g., Richards et al., 2011), and uncharacterized blending in the red wing of the line (i.e., the "red shelf"; see Fine et al., 2010) have led some to believe that there are unacceptably large uncertainties and systematic biases in $\mathrm{C}$ IV-based masses, compared with $\mathrm{H} \beta$ (e.g., Baskin \& Laor, 2005; Netzer et al., 2007; Shen et al., 2008). On the other hand, other studies have found consistent agreement between C IV and H $\beta$ masses (e.g., Vestergaard, 2002; 2004; Warner et al., 2003; Kollmeier et al., 2006; Greene et al., 2010). We suspect that much of the observed inconsistency found between these studies lies in the quality of the high-z data typically used for these investigations, as discussed above. We have specifically shown for $\mathrm{H} \beta$ that systematic biases in line widths and $\mathrm{BH}$ masses increase as data quality is degraded (Denney et al., 2009a). In addition, Vestergaard \& Peterson (2006) have argued that $S / N$ and absorption accounts for much of the discrepancy between their results and those of Baskin \& Laor (2005) for PG quasars.

Greene et al. (2010) recently showed that there was no systematic bias (though still a large dispersion of $\sim 0.5$ dex) between $\mathrm{C}$ IV and $\mathrm{H} \beta$ based $\mathrm{BH}$ masses with a sample of high redshift, lensed QSOs from the CASTLES survey (Falco et al. 2001). However, because they targeted only the highest luminosity objects in the sample, they were limited to a small range in BH mass, and their study could not address whether or not there are any mass-dependent biases between these two estimates. We extended this sample with additional IR spectroscopic observations (Assef et al. 2010) of $\mathrm{H} \beta$, and, equally important, we gathered high $S / N$ C IV spectra, either from the literature or from new observations, for all targets to reduce the possibility of any biases resulting from low quality data and/or inconsistent line-width measurement techniques. Not only did we extend the mass range compared to Greene et al., but our analysis uncovered a statistically significant correlation between the $\mathrm{C}$ IV to $\mathrm{H} \beta$ mass residuals and the ratio of $\mathrm{UV}$ to optical luminosity. 


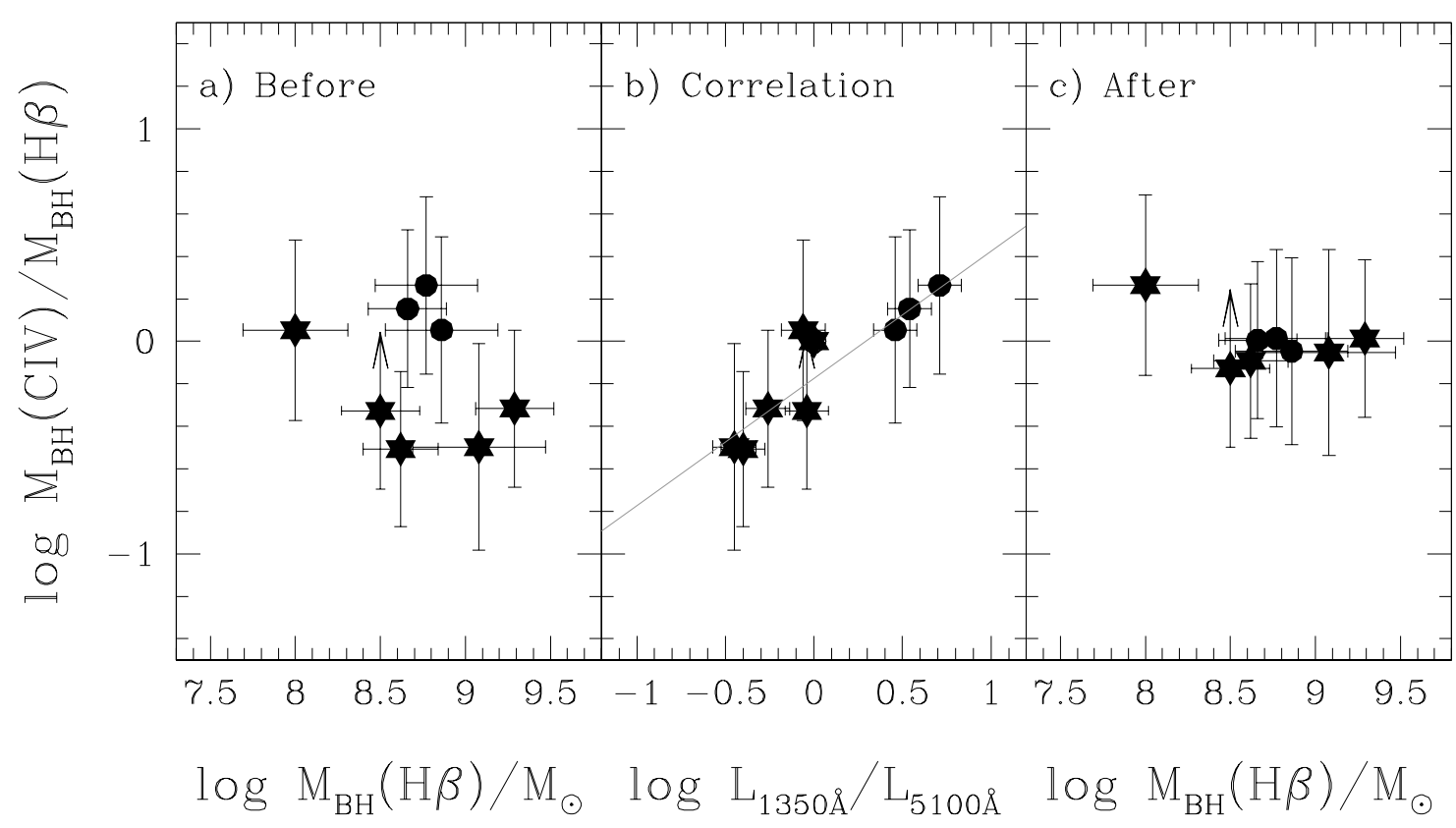

Figure 5: Left panel shows the $\mathrm{C}$ IV to $\mathrm{H} \beta$ mass residuals before the color correction, the middle panel shows the correlation of the mass residuals with the UV to optical luminosity ratio, and the right panel shows the mass residuals after the color correction.

Such a correlation may be expected due to reddening, host contamination or non-universal AGN SEDs, but these would produce a different correlation slope than what we find, unless these effects are correlated with the broad emission line widths (see Assef et al. for details). After correcting the C IV-based masses for this color bias, the dispersion in the mass residuals was decreased by a factor of $\sim 2$ (the exact decrease is dependent on the line width characterization used for the mass estimate), showing $\mathrm{C}$ IV and $\mathrm{H} \beta$ masses to be highly consistent for our sample. If nothing else, it is clear that much of the scatter between $\mathrm{H} \beta$ and $\mathrm{C}$ IV BH mass estimates is due to inconsistencies in the continuum luminosity estimates rather than the line widths. Figure 5, adapted from Assef et al., shows an example of these results for C IV masses measured by characterizing the line width through the line dispersion, $\sigma_{l}$, and $\mathrm{H} \beta$ masses using the FWHM. Application of this correction to other samples of objects also shows a decrease in the scatter between $\mathrm{C}$ IV and $\mathrm{H} \beta$ masses, suggesting that with high quality observations and this correction, $\mathrm{BH}$ masses can be reliably estimated at all redshifts with C IV observations.

\section{Summary}

As a result of technological advancements in telescopes, computers, and data analysis software, observational and statistical uncertainties should no longer be the largest source of uncertainty in the types of measurements necessary for measuring BH masses. Using low $S / N$ data introduces both statistical and systematic uncertainties and can be avoided only by using high quality data. These systematic uncertainties dominate the error budget. We have shown here that these systematics enter into mass measurements through physical properties of the systems being observed (e.g., absorption), as well as both inherent and resource-dependent observational constraints 
(e.g., line-of-sight orientation and time resolution) in making the measurements necessary for fully understanding BHs and their accretion. Our efforts to identify and mitigate these uncertainties are starting to pay off, as we recognize and determine ways to correct for various sources of systematics. If we want an accurate census of black holes and their growth in order to reliably investigate their evolution and their possible coevolution with galaxies over cosmic times, it is imperative that the community widely acknowledge and actively work to mitigate these and other systematic uncertainties in $\mathrm{BH}$ mass measurements.

\section{References}

[1] R. Antonucci, Unified models for active galactic nuclei and quasars, Annual Review of Astronomy and Astrophysics 31 (1993) 473-521.

[2] R. J. Assef et al., Black Hole Mass Estimates Based on CIV are Consistent with Those Based on the Balmer Lines, ArXiv e-prints (Sept., 2010) [1009.1145].

[3] A. Baskin and A. Laor, What controls the CIV line profile in active galactic nuclei?, MNRAS 356 (Jan., 2005) 1029-1044, [arXiv: astro-ph/0 409196 ].

[4] M. C. Bentz, B. M. Peterson, H. Netzer, R. W. Pogge, and M. Vestergaard, The Radius-Luminosity Relationship for Active Galactic Nuclei: The Effect of Host-Galaxy Starlight on Luminosity Measurements. II. The Full Sample of Reverberation-Mapped AGNs, ApJ 697 (May, 2009a) 160-181, [0812.2283].

[5] M. C. Bentz et al., The Lick AGN Monitoring Project: Broad Line Region Radii and black Hole Masses from Reverberation Mapping of H $\beta$, ApJ 705 (Nov., 2009b) 199-217, [0 908.0003 ].

[6] M. C. Bentz et al., The Lick AGN Monitoring Project: Velocity-delay Maps from the Maximum-entropy Method for Arp 151, ApJL 720 (Sept., 2010) L46-L51.

[7] R. D. Blandford and C. F. McKee, Reverberation mapping of the emission line regions of Seyfert galaxies and quasars, ApJ 255 (Apr., 1982) 419-439.

[8] J. Debuhr, E. Quataert, and C.-P. Ma, The growth of massive black holes in galaxy merger simulations with feedback by radiation pressure, MNRAS 412 (Apr., 2011) 1341-1360, [1006 . 3312].

[9] K. D. Denney, B. M. Peterson, M. Dietrich, M. Vestergaard, and M. C. Bentz, Systematic Uncertainties in Black Hole Masses Determined from Single Epoch Spectra, ApJ 692 (Feb., 2009a) 246-264, [0810.3234].

[10] K. D. Denney et al., A Revised Broad-Line Region Radius and Black Hole Mass for the Narrow-Line Seyfert 1 NGC 4051, ApJ 702 (Sept., 2009b) 1353-1366, [0 904 . 0251].

[11] K. D. Denney et al., Diverse Broad Line Region Kinematic Signatures From Reverberation Mapping, ApJL 704 (Oct., 2009c) L80-L84, [0 908 . 0327].

[12] K. D. Denney et al., Reverberation Mapping Measurements of Black Hole Masses in Six Local Seyfert Galaxies, ApJ 721 (Sept., 2010) 715-737, [1006.4160].

[13] E. E. Falco et al., The CASTLES Gravitational Lensing Tool, (T. G. Brainerd \& C. S. Kochanek, ed.), vol. 237 of Astronomical Society of the Pacific Conference Series, pp.25+, 2001.

[14] L. Ferrarese and D. Merritt, A Fundamental Relation between Supermassive Black Holes and Their Host Galaxies, ApJL 539 (Aug., 2000) L9-L12. 
[15] S. Fine, S. M. Croom, J. Bland-Hawthorn, K. A. Pimbblet, N. P. Ross, D. P. Schneider, and T. Shanks, The CIV linewidth distribution for quasars and its implications for broad-line region dynamics and virial mass estimation, MNRAS 409 (Dec., 2010) 591-610, [10 05 . 5287].

[16] K. Gebhardt et al., A Relationship between Nuclear Black Hole Mass and Galaxy Velocity Dispersion, ApJL 539 (Aug., 2000) L13-L16.

[17] J. E. Greene, C. Y. Peng, and R. R. Ludwig, Redshift Evolution in Black Hole-Bulge Relations: Testing C IV-Based Black Hole Masses, ApJ 709 (Feb., 2010) 937-949, [0911 . 0685 ].

[18] K. Gültekin et al. The M- $\sigma$ and M-L Relations in Galactic Bulges, and Determinations of Their Intrinsic Scatter, ApJ 698 (June, 2009) 198-221, [0 903 . 4897].

[19] N. Häring and H. Rix, On the Black Hole Mass-Bulge Mass Relation, ApJL 604 (Apr., 2004) L89-L92, [arXiv:astro-ph/0402376].

[20] P. F. Hopkins and M. Elvis, Quasar feedback: more bang for your buck, MNRAS 401 (Jan., 2010) 7-14, [0 904 . 0649 ].

[21] K. Horne, Echo Mapping Problems Maximum Entropy solutions, in Reverberation Mapping of the Broad-Line Region in Active Galactic Nuclei (P. M. Gondhalekar, K. Horne, \& B. M. Peterson, ed.), vol. 69 of Astronomical Society of the Pacific Conference Series, pp. 23-25, 1994.

[22] K. Horne, B. M. Peterson, S. J. Collier, and H. Netzer, Observational Requirements for High-Fidelity Reverberation Mapping, PASP 116 (May, 2004) 465-476.

[23] K. Jahnke and A. Maccio, The non-causal origin of the black hole-galaxy scaling relations, ArXiv e-prints (June, 2010) [1006.0482].

[24] S. Kaspi, P. S. Smith, H. Netzer, D. Maoz, B. T. Jannuzi, and U. Giveon, Reverberation Measurements for 17 Quasars and the Size-Mass-Luminosity Relations in Active Galactic Nuclei, ApJ 533 (Apr., 2000) 631-649.

[25] J. A. Kollmeier et al., Black Hole Masses and Eddington Ratios at $0.3<z<4$, ApJ 648 (Sept., 2006) 128-139, [arXiv:astro-ph/0508657].

[26] C. Y. Kuo et al., The Megamaser Cosmology Project. III. Accurate Masses of Seven Supermassive Black Holes in Active Galaxies with Circumnuclear Megamaser Disks, ApJ 727 (Jan., 2011) 20-+, [1008.2146].

[27] A. Marconi and L. K. Hunt, The Relation between Black Hole Mass, Bulge Mass, and Near-Infrared Luminosity, ApJL 589 (May, 2003) L21-L24, [arXiv : astro-ph / 0304274 ].

[28] S. Mathur, Narrow-line Seyfert 1 galaxies and the evolution of galaxies and active galaxies, MNRAS 314 (June, 2000) L17-L20, [arXiv : astro-ph/0003111].

[29] J. Moran, L. Greenhill, J. Herrnstein, P. Diamond, M. Miyoshi, N. Nakai, and M. Inque, Probing Active Galactic Nuclei with H_2O Megamasers, Proceedings of the National Academy of Science 92 (Dec., 1995) 11427-11433.

[30] H. Netzer, P. Lira, B. Trakhtenbrot, O. Shemmer, and I. Cury, Black Hole Mass and Growth Rate at High Redshift, ApJ 671 (Dec., 2007) 1256-1263, [0 08 . 3787].

[31] C. A. Onken, L. Ferrarese, D. Merritt, B. M. Peterson, R. W. Pogge, M. Vestergaard, and A. Wandel, Supermassive Black Holes in Active Galactic Nuclei. II. Calibration of the Black Hole Mass-Velocity Dispersion Relationship for Active Galactic Nuclei, ApJ 615 (Nov., 2004) 645-651. 
[32] C. Y. Peng, How Mergers May Affect the Mass Scaling Relation between Gravitationally Bound Systems, ApJ 671 (Dec., 2007) 1098-1107, [0 704 . 1860].

[33] B. M. Peterson, Reverberation mapping of active galactic nuclei, PASP 105 (Mar., 1993) 247-268.

[34] B. M. Peterson et al., X-Ray and Optical Variability in NGC 4051 and the Nature of Narrow-Line Seyfert 1 Galaxies, ApJ 542 (Oct., 2000) 161-174.

[35] B. M. Peterson et al., Central Masses and Broad-Line Region Sizes of Active Galactic Nuclei. II. A Homogeneous Analysis of a Large Reverberation-Mapping Database, ApJ 613 (Oct., 2004) 682-699.

[36] A. Rafiee and P. B. Hall, Biases in the Quasar Mass-Luminosity Plane, ArXiv e-prints (Nov., 2010) [1011.1268].

[37] G. T. Richards et al., Unification of Luminous Type 1 Quasars through C IV Emission, AJ 141 (May, 2011) 167+, [1011 .2282].

[38] Y. Shen, J. E. Greene, M. A. Strauss, G. T. Richards, and D. P. Schneider, Biases in Virial Black Hole Masses: An SDSS Perspective, ApJ 680 (June, 2008) 169-190, [arXiv : 0709. 3098].

[39] M. Vestergaard, Determining Central Black Hole Masses in Distant Active Galaxies, ApJ 571 (June, 2002) 733-752.

[40] M. Vestergaard, Early Growth and Efficient Accretion of Massive Black Holes at High Redshift, ApJ 601 (Feb., 2004) 676-691.

[41] M. Vestergaard and B. M. Peterson, Determining Central Black Hole Masses in Distant Active Galaxies and Quasars. II. Improved Optical and UV Scaling Relationships, ApJ 641 (Apr., 2006) 689-709, [arXiv:astro-ph/0601303].

[42] C. Warner, F. Hamann, and M. Dietrich, A Relation between Supermassive Black Hole Mass and Quasar Metallicity?, ApJ 596 (Oct., 2003) 72-84, [arXiv: astro-ph/ 0307247 ].

[43] J. Woo et al., The Lick AGN Monitoring Project: The $M_{\mathrm{BH}}-\sigma_{\star}$ Relation for Reverberation-mapped Active Galaxies, ApJ 716 (June, 2010) 269-280, [1004 . 0252]. 